\title{
Evaluation de la prise en charge de la maternité sans risque et de la survie de l'enfant au niveau communautaire de Bazéga
}

Direction de la Santé et de la Famille (DSF)

University of Ouagadougou Demographic Research Unit (UERD)

Mwangaza

Population Council

\section{Recommended Citation}

"Evaluation de la prise en charge de la maternité sans risque et de la survie de l'enfant au niveau communautaire de Bazéga," Final report. Washington, DC and Bazega: Population Council and Laboratoire de Santé Communautaire, 1998. 


\title{
LABORATOIRE DE SANTE COMMUNAUTAIRE, BAZEGA
}

\author{
EVALUATION DE LA PRISE EN CHARGE DE LA \\ MATERNITE SANS RISQUE ET DE LA SURVIE DE \\ L'ENFANT AU NIVEAU COMMUNAUTAIRE DU BAZEGA
}

MINISTERE DE LA SANTE

Secrétariat Général

Direction Générale de la Santé Publique

Direction de la Santé de la Famille
Unité d'Enseignement et de Recherche en Démographie
Mwangaza

Population Council Africa OR/TA II

SERIE DOCUMENTAIRE No 20

Ouagadougou, Burkina Faso Décembre 1998 


\section{TABLE DES MATIERES}

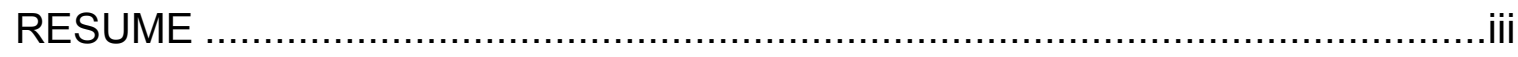

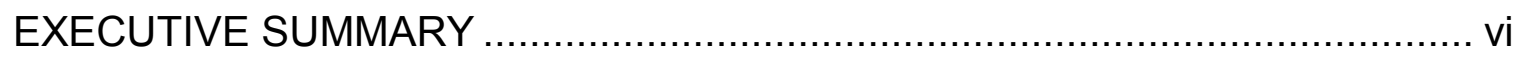

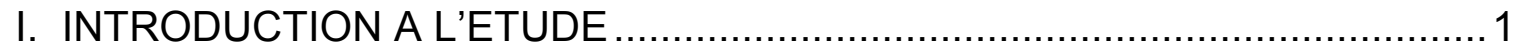

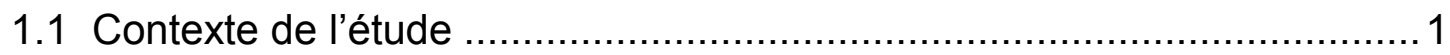

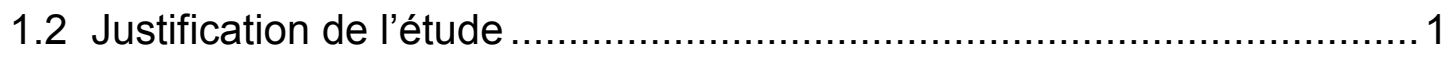

1.3 Objectifs de l'étude et questions de recherche .................................... 3

1.3.1 Objectifs ........................................................... 3

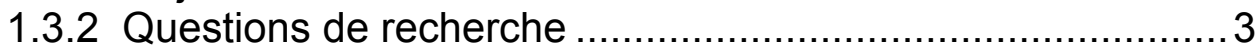

1.4 Approche méthodologique ......................................................

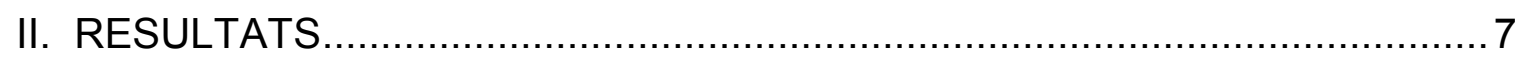

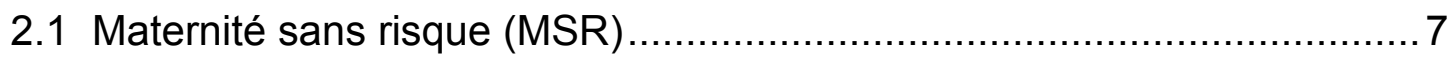

2.1.1 Connaissances, perception, et comportement ...................

2.1.2 Implication des hommes.............................................. 15

2.1.3 Implication des agents SBC dans les prestatations de services de maternité sans risque et de survie de l'enfant. 16

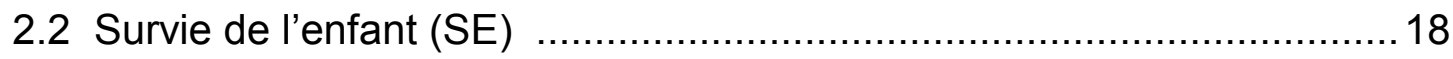

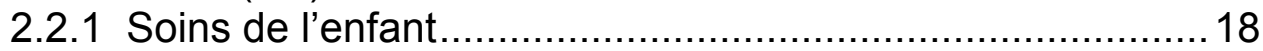

2.2.2 Nutrition de l'enfant ...................................................... 19

2.2.3 Rites et coutumes relatifs à la survie de l'enfant ................ 19

III. RESULTATS DES ATELIERS D'AUTO-ANALYSE ................................20

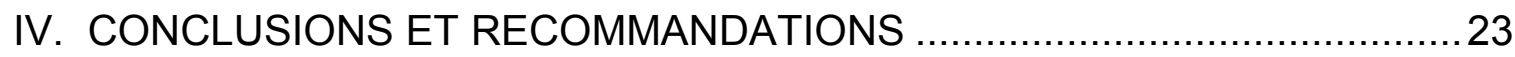




\section{TABLEAU DES SIGLES ET ABREVIATIONS}

$\begin{array}{ll}\text { AAA } & \text { Atelier d'auto-analyse } \\ \text { ASC } & \text { Agent de la santé communautaire } \\ \text { CSPS } & \text { Centre de la santé et de la promotion sociale } \\ \text { DSF } & \text { Direction de la santé de la famille } \\ \text { EDS } & \text { Enquête démographique et de santé } \\ \text { EIA } & \text { Entretien individuel approfondi } \\ \text { FS } & \text { Formation sanitaire } \\ \text { GDD } & \text { Groupe discussion dirigée } \\ \text { LSC } & \text { Laboratoire de santé communautaire } \\ \text { MSR } & \text { Maternité sans risque } \\ \text { ONG } & \text { Organisation non-gouvernementale } \\ \text { PF } & \text { Planification familiale } \\ \text { SBC } & \text { Services à base communautaire } \\ \text { SE } & \text { Survie de l'enfant } \\ \text { UERD } & \text { Unité d'enseignement et de recherche en démographie }\end{array}$




\section{RESUME}

\section{Introduction}

Cette étude vient à point nommé pour fournir des informations sur les actions actuelles aux niveaux communautaires et individuels en matière de survie de l'enfant et de maternité sans risque. Les prestataires et le Ministère de la Santé, ainsi que tout intervenant, pourront par conséquent utiliser les résultats pour l'élaboration d'interventions ou actions appropriées. C'est une étape préalable au développement de stratégies idoines en matière de survie de l'enfant et maternité sans risque dans le cadre du Laboratoire de Santé Communautaire (LSC).

Aussi bien en ville qu'en campagne, la nécessité d'avoir une meilleure compréhension de la perception, par les communautés et les prestataires communautaires, des signes précurseurs de risque pendant la grossesse, l'accouchement et le post-partum, l'itinéraire thérapeutique ou la prise en charge médicale ou communautaire des femmes enceintes et accouchées ainsi que les activités en matière de survie de l'enfant, s'impose, surtout quand on veut élaborer des stratégies culturellement acceptables. C'est dans cet esprit qu'il a été commandité une étude qualitative pour contribuer à l'élaboration d'interventions communautaires appropriées en matière de survie de l'enfant et de maternité sans risque dans la zone du LSC.

\section{Méthodologie}

La présente étude a nécessité l'utilisation de trois approches méthodologiques en recherche qualitative pour la collecte et l'analyse des données dans les zones concernées par l'étude. Ce sont les groupes de discussions dirigées, les entretiens individuels approfondis et enfin des ateliers d'auto-analyse avec les membres des comités de santé et les leaders communautaires. Au niveau de la communauté, cinq groupes cibles étaient concernés : les adolescents, garçons comme filles de 15 à 19 ans, les femmes et hommes mariés de 20 à 29 ans et de 30 à 49 ans ayant un enfant de moins de cinq ans, et les femmes de plus de 49 ans communément appelées "Yaaba" (en langue nationale Mooré) ou grand-mères. En somme, 14 groupes de discussion dirigées (GDD) ont été menés. Quant aux interviews individuelles approfondies, 16 ont été conduites avec les agents de services à base communautaire, les accoucheuses villageoises et les tradithérapeutes. Deux ateliers d'auto-analyse ont été réalisés avec les membres des comités de gestion, les prestataires des formations sanitaires et les membres de l'équipe cadre des deux districts sanitaires de la zone d'étude.

\section{Résultats clés}

Des signes physiques, sympathiques et l'abandon de certaines activités sociales et religieuses sont cités pour dire qu'une femme est en période de grossesse. En terme de communication, pour annoncer à son mari qu'elle est enceinte, la femme utilise un langage consacré et codifié. La grossesse est en priorité annoncée au mari, à la belle-mère, à une meilleure amie, et en dernière position à la co-épouse.

Tout au long de sa grossesse, la femme est soumise à des interdits et recommandations alimentaires dans le but d'éviter les complications ou que le bébé prenne trop de poids. II en est de même sur le plan thérapeutique. Ainsi, le miel est interdit car il serait abortif. Par contre il lui est recommandé de bien manger dans la 
mesure où elle mange pour deux et de manger des aliments qui lui donnent "du sang et de la force."

Pour ce qui concerne les rapports sexuels pendant la grossesse, deux tendances se dégagent : ceux qui estiment que les rapports devraient continuer modérément et ceux qui pensent qu'il est interdit.

Les causes de complications pendant la grossesse et l'accouchement sont perçus de manière diverse: une grossesse précoce, l'inactivité prolongée, et l'infidélité. Pendant la grossesse, les douleurs au bas ventre, la petite taille, les vomissements et les œdèmes des pieds sont cités comme signes de reconnaissance de complications. Pendant l'accouchement, c'est surtout une mauvaise présentation du bébé, un travail prolongé et la rétention du placenta qui sont reconnus comme signes de complication.

La prise en charge des complications est faite de plusieures façons selon la cause. D'abord, certains fatalistes se réfèrent à la prière et aux sacrifices pour obtenir la levée de la complication. Des rites et coutumes sont souvent pratiqués. Cependant il est important de noter qu'il est souvent fait référence en priorité aux formations sanitaires aux agents SBC et enfin aux accoucheuses villageoises pour obtenir des conseils pendant la grossesse et le post-partum. Par contre quand il s'agit des soins, la priorité est d'abord offerte aux formations sanitaires, aux accoucheuses villageoises et enfin aux agents SBC.

En ce qui concerne l'implication des hommes, il ressort que les rôles reconnus appropriés ou exigés de l'homme-de soutien physique et moral--ne sont pas toujours exécutés comme il se doit par celui-ci. La communication n'existe pratiquement pas entre l'homme et son épouse/partenaire pendant la grossesse et le post-partum. Le rôle de l'homme tel que vécu actuellement est lié au phénomène culturel et social qui le lui confère, donc au genre. Le rôle de l'homme pendant la grossesse, l'accouchement et le post-partum s'articule autour de l'achat des médicaments, de la provision des aliments. A l'unanimité, les femmes souhaitent vivement que cela change.

L'un des objectifs de cette étude était aussi de savoir si la communauté approuverait l'utilisation des agents SBC pour mener des activités en matière de suivi des femmes en grossesse et du post-partum. Unanimement, les enquêtés acceptent cette éventualité. Le sexe de l'agent SBC ne semble pas avoir d'importance à l'exception des adolescents qui pensent que les femmes doivent parler aux femmes et les hommes aux hommes. Le souhait général est de donner des conseils aux couples ensemble au même moment.

Les soins, ainsi que la nutrition des enfants sont surtout effectués en accord avec les pratiques traditionnelles: il s'agit des gavages et des purges intestinales auxquels l'on reconnaît des vertus thérapeutiques. Cependant, certaines personnes interviewées mentionnent la vaccination.

\section{Conclusion et recommandations}

Les résultats de cette étude sont importants pour les décideurs et les intervenants en matière de maternité sans risque et de survie de l'enfant. II s'agit de données 
qualitatives donnant la perception des membres de la communauté. C'est une étape importante à l'élaboration de stratégies d'intervention appropriées en matière de survie de l'enfant et de maternité sans risques. Une dissémination des résultats clefs serait nécessaire pour les prestataires de services cliniques et communautaires, ce qui leur permettrait de réorienter leur stratégie en matière de communication en l'endroit des hommes et des femmes fréquentant leur formation sanitaire. De plus, il s'avère que les agents SBC peuvent être des relais importants pour les prestations de services à l'endroit des femmes enceintes et accouchées. Des efforts sont à faire dans le cadre de la participation des hommes de manière holistique aux côtés de leur épouses/partenaires aux services de santé de la reproduction.

Les agents SBC sont vus comme des potentialités pouvant contribuer à l'augmentation des connaissances, attitudes et pratiques des communautés vis à vis de la maternité sans risque et de la survie de l'enfant. Impliquer l'homme dans les sessions de counseling est bien accueilli par la majorité des enquêtés. Ces sessions de counseling devraient intégrer des discussions sur les raisons pour une femme enceinte d'éviter les travaux durs, et ce, dans le but de combler le fossé entre le rôle de l'homme souhaité par la femme et son rôle actuel.

Les adolescents devraient avoir un counseling particulier dans la mesure où ils pensent qu'une femme devrait mener un counseling auprès d'une femme et un homme auprès d'un homme: les agents SBC de sexe masculin devrait causer avec les hommes et ceux de sexe féminin avec les femmes. La vaccination devrait être plus tard intégrée dans le paquet de services des agents SBC.

Enfin toutes ces leçons apprises devraient être prises en compte pour le développement de stratégies d'intervention pour l'amélioration de la morbidité et de la mortalité maternelles et infantiles au Bazèga. 


\section{EXECUTIVE SUMMARY}

\section{Introduction}

This qualitative study was undertaken to develop an understanding of perceptions and behaviors regarding safe motherhood and child survival in the rural community of Bazèga, Burkina Faso, and to explore the acceptability of involving communitybased distribution (CBD) workers in activities centered around these issues. This will serve as the first step in developing intervention strategies for safe motherhood and child survival programs for the Laboratoire de Santé Communautaire (LSC), or community health research laboratory in Bazèga.

A strong understanding is needed of both communities' and health providers' perceptions of maternal health-warning signs during pregnancy, delivery and postpartum and how these signs of complications are managed and treated-as well as perceptions of child survival, if culturally acceptable intervention strategies are to be developed. This study provides such an understanding of perceptions in Bazèga.

\section{Methodology}

Three qualitative methodological tools were used: focus group discussions, individual in-depth interviews and self-analysis workshops. Focus group participants were divided by sex and age into seven categories-boys and girls between 15 and 19 years old, married men and women between 20 and 29 or 30 and 49, with at least one child under 5 years old, and "yaaba," or grandmothers over 49 . These groups were identified in communities surrounding two health centers, Rakaye and Komsilga, resulting in 14 focus groups altogether. Individual interviews were held with 16 health workers, including health providers from the health centers, traditional practictioners, community-based distribution workers and village birth attendants. Finally, self-analysis workshops were held with community leaders and health center staff to validate the results.

\section{Key findings}

Community members recognized that there are signs indicating pregnancy. The main signs mentioned were physical changes, particularly gaining or losing weight and lightening of skin, as well as behavioral changes, such as stopping certain daily chores or religious activities. Husbands and mothers-in-law are the first people a woman generally tells she is pregnant, often using a codified language, such as, "I am not the same as I was yesterday."

To avoid complications and prevent the baby from gaining too much weight, women are expected to follow certain dietary recommendations. For example, honey is considered an abortifacient and is to be avoided, while foods such as eggs, fish and meats are considered beneficial because they "give blood" to the woman.

In terms of coummunity attitudes toward sexual relations during pregnancy, there are varying opinions. Some community members feel sex should stop after the third or fourth month, while others feel it is permissible in moderation until just before the woman gives birth.

Similarly, community members cited a variety of causes of complications during pregnancy and delivery. These included prolonged inactivity, infidelity, and 
adolescent pregnancy. Signs of complications during pregnancy mentioned were pain in the lower abdomen, vomiting and a rash or edema on the feet. Women who are small in stature were also considered at risk for complications. During the delivery, malpositioning of the fetus, prolonged labor, and retention of the placenta were all recognized as complications.

Different courses of action to handle complications were cited. For some, prayer, sacrifices and rituals were the best recourse. The majority, however, recommended going to the health center, traditional birth attendants or community-based distribution workers as ways of handling complications. Community members reported that CBD workers were more helpful than tradiational birth attendants in terms of counseling and advice, while traditional birth attendants were more appropriate than CBD workers for actual delivery of health care services.

Both men and women described men's role primarily in terms of economic support both during and after pregnancy. Men are considered responsible for providing food and medication for their partners. Respondents also mentioned that men needed to help with women's labor-intensive chores, such as fetching water and collecting wood. However, this was usually mentioned as a desirable role for men, one which is not often practiced. Women generally expressed a desire for men's roles to change. In addition, people felt there was little communication between spouses during pregnancy and the post-partum period.

Community members felt it would be acceptable for CBD workers to provide counseling on safe motherhood. Interestingly, the majority of those interviewed did not feel that the sex of the CBD worker was important. Furthermore, they expressed the desire to be counseled as a couple. Adolescents were an exception: they felt that women should counsel women, and men should counsel men.

When asked about child nutrition and care, traditional practices were most frequently mentioned, including intestinal purging and overfeeding the child. However, a few respondents mentioned vaccinations.

\section{Conclusions and Recommendations}

The results of this study are important for decision makers and those designing interventions for safe motherhood and child survival. As a qualitative study of the perceptions of community members and health professionals, it may serve as the first step to designing appropriate interventions. A dissemination of key findings will be held for clinical and community health workers to allow them to interpret the results and translate the implications into changes in their day to day interactions with clients.

Community-based distribution workers are seen as a viable tool for increasing knowledge on safe motherhood and child survival issues and practices. Furthermore, involving men during these counseling sessions is regarded favorably by the majority of the community. In order to help bridge the gap between men's desired role and their actual role, these sessions should include discussions of why pregnant women should not perform labor-intensive tasks. Adolescents may require special counseling and efforts to increase communication in their relationships, as evidenced by their reluctance to be counseled as a couple and their preference to be 
counseled by same-sex CBD workers. Finally, vaccination of children needs to be further stressed as an integral part of child survival, possibly by integrating promotion of these services into the package provided by CBD workers. All of these findings will need to be taken into account when developing an intervention strategy to improve maternal and infant morbidity and mortality in Bazèga. 


\section{INTRODUCTION A L'ETUDE}

\subsection{Contexte de l'étude}

La mortalité maternelle demeure très élevée dans une grande partie du monde avec 585.000 décès chaque année dont $99 \%$ surviennent dans les pays en voie de développement. Au Burkina Faso, le taux de mortalité maternelle varie selon les sources, entre 500 et 600 pour 100.000 naissances vivantes. ${ }^{1}$ Les principales causes de décès sont les hémorragies pendant le travail ou après l'accouchement, les infections et les fausses couches.

Ces statistiques illustrent très clairement les énormes problèmes de santé auxquels les femmes sont confrontées par rapport à l'accouchement sans risque et à l'assurance de la survie des enfants au-delà des cinq années de vie. De façon manifeste, les services de santé ne touchent pas les populations auxquelles ils étaient destinés pour résoudre ses problèmes. Généralement en Afrique subsaharienne, et plus particulièrement dans la région du Sahel, la prise en charge des problèmes de santé de la femme enceinte et des bébés et enfants par les femmes et les familles est très peu comprise ou même totalement ignorée. Cependant il se constitue un ensemble de connaissances sur comment les urgences obstétricales sont prises en charge, et sur comment les populations rurales ayant difficilement accès aux services sanitaires prennent en charge les problèmes de santé des nouveau-nés et des enfants de moins de cinq ans.

Une collecte de données de base est la première étape de résolution d'un problème de santé publique. Aussi l'objectif de cette étude est de collecter des données de base permettant de comprendre les perceptions, les connaissances, les itinéraires thérapeutiques de la communauté vis-à-vis de la maternité sans risque et des soins accordés aux enfants de moins de cinq ans.

\subsection{Justification de l'étude}

Reconnaissant la gravité de ces problèmes, le Ministère de la Santé du Burkina Faso a mis au point un programme de maternité sans risque qui a débuté en 1996 et qui est présentement en cours. ${ }^{2}$

Dans sa phase initiale, la collecte de données de base est à la fois nécessaire et pertinente pour l'élaboration de stratégies culturellement acceptables qui correspondent aux besoins des communautés. D'après l'enquête démographique de 1993, près de $40 \%$ des femmes au niveau national ne reçoivent pas au moins une consultation prénatale pendant la grossesse, bien que dans la région du Bazèga ce taux ne soit que de $10 \%{ }^{3}$. II n'existe pas de statistiques permettant d'évaluer le niveau de compréhension des signes de danger pendant une grossesse et un travail difficiles. Immédiatement après l'accouchement et pendant la période post-partum de récupération, la femme a besoin de beaucoup d'informations sur différents aspects de sa santé et de celle de son nouveau-né. Cependant, les examens postnatals par les femmes fréquentant les formations sanitaires ou par le personnel

1 Enquête Démographique et de Santé (EDS), Burkina Faso, 1993.

2 Programme de Maternité Sans Risques, Document Préliminaire, Burkina Faso, 1996.

3 Enquête Démographique et de Santé (EDS), Burkina Faso, 1993. 
médical visitant les femmes sont très rares au Burkina Faso aussi bien en zone rurale qu'urbaine.

La mortalité infantile est estimée autour de 120 pour 1000 naissances vivantes tandis que le taux de mortalité des nourrissons et des enfants était de 202 pour 1000 dans la zone de l'étude du Bazèga. ${ }^{4}$ Le paludisme et la diarrhée apparaissent comme les problèmes majeurs de santé chez les enfants dans le Bazèga, avec $17 \%$ des enfants qui souffraient de paludisme les deux dernières semaines et $22 \%$ qui souffraient de diarrhée au moment de l'enquête de base. ${ }^{5}$ A cause des pratiques traditionnelles, pratiquement aucun nouveau-né ne bénéficie du colostrum de sa mère parce qu'il n'est pas accepté que cela soit donné aux bébés. Deux bébés sur trois ont été partiellement ou pas du tout vaccinés contre le tétanos. Ces statistiques démontrent clairement les problèmes de survie des enfants aussi bien au Bazèga qu'au niveau national.

Pour ces raisons, la Direction de la Santé de la Famille (DSF) se propose d'entreprendre une étude prospective sur les connaissances et les perceptions des problèmes de santé maternelle et infantile, et sur les pratiques actuelles de prise en charge des populations vivant dans la zone d'étude du Laboratoire de Santé Communautaire (LSC) du Bazèga. ${ }^{6}$

L'objet de cette étude est de travailler avec les communautés de la zone pour identifier les problèmes de santé auxquels elles sont le plus fréquemment confrontées, comprendre comment ces problèmes sont actuellement pris en charge, et identifier les informations nécessaires pour avoir les comportements élémentaires et de routine de recherche de la santé qui manquent en ce moment. Sur la base de ces informations les populations seront invitées à suggérer des interventions adaptées, sur le plan culturel et faisables sur le plan programmatique, qui pourraient renforcer les services de maternité sans risque et de survie de l'enfant disponibles dans la zone. Une attention toute particulière a été accordée à la faisabilité de l'utilisation des agents de services à base communautaire (SBC) nouvellement introduits, des agents de santé communautaire comme les accoucheuses villageoises, les accoucheuses traditionnelles, les tradithérapeutes ainsi que les comités de gestion de santé.

\footnotetext{
${ }^{4}$ Rapport de l'enquête quantitative de base, Laboratoire de Santé Communautaire (LSC), Burkina Faso, 1997.

${ }^{5}$ Idem.

6 Dans l'optique d'améliorer les actions en matière de SR/PF, le Ministère de la Santé à travers la Direction de la Santé et de la Famille (DSF) en collaboration avec l'Université de Ouagadougou, à travers l'Unité d'Enseignement et de Recherche en Démographie (UERD), Mwangaza et le Projet de Recherche Opérationnelle et Assistance Technique en Afrique II (OR/TA II) du Population Council a entrepris la mise en place d'un Laboratoire de Santé Communautaire (LSC) dans la province du Bazèga dont le mandat est de tester des approches de santé reproductive au niveau communautaire avant leur extension au niveau national.
} 


\subsection{Objectifs de l'étude et questions de recherche}

\subsubsection{Objectifs}

\section{a) Objectif général}

L'objectif final est d'identifier des interventions acceptables, faisables et durables qui contribueront à la réduction de la morbidité et de la mortalité maternelles et infantiles.

\section{b) Objectifs immédiats}

L'étude a visé les objectifs immédiats suivants :

- Explorer les connaissances et les perceptions des membres des communautés sur des besoins en informations et en services des femmes enceintes, des signes de complication pendant le travail, les noms de ces complications en langue locale et les actions à entreprendre face à ces signes de danger.

- Identifier les connaissances, les perceptions, les normes sociales et l'itinéraire thérapeutique individuel, au niveau des familles et de la communauté dans la période suivant l'accouchement.

- Evaluer l'acceptabilité et la faisabilité de l'utilisation des différents types d'agents communautaires pour offrir des informations et des services de santé aux femmes pendant la grossesse et après l'accouchement.

- Explorer le rôle joué par les hommes dans les soins de santé au profit des femmes enceintes et accouchées (accouchement, période post-partum) et des enfants durant les cinq premières années de leur vie .

- Proposer des interventions à base communautaire qui soutiendront et éduqueront les femmes et les enfants pendant la grossesse, l'accouchement et la période suivant l'accouchement.

\subsubsection{Questions de recherche}

Cette étude se propose de répondre aux questions essentielles suivantes :

a) Quelles sont les connaissances, les perceptions et les comportements de la communauté vis-à-vis des besoins des femmes enceintes et des signes de complication pendant l'accouchement?

- Quelles actions les membres de la communauté entreprennent-ils pendant les urgences survenant au cours de l'accouchement?

- Quels noms donnent-ils aux signes de danger apparaissant pendant l'accouchement? Y a-t-il des signes de complications auxquels l'on doit s'attendre pendant le travail qui sont reconnaissables pendant la grossesse ? 
b) Quels sont les connaissances, les perceptions et les comportements les plus répandus par rapport aux soins suivant l'accouchement au niveau de la communauté (des hommes et des femmes)?

c) Quels rôles les hommes jouent-ils pendant la grossesse, à l'accouchement et après l'accouchement? S'ils n'ont aucun rôle à jouer, pourquoi en est-il ainsi ?

d) Que pensent les membres de la communauté du recours aux agents communautaires pendant la grossesse, pour l'accouchement et dans la période suivant l'accouchement pour offrir des informations et des services y afférents ? Le sexe de l'agent SBC joue-t-il un rôle déterminant ? Si tel est le cas, pourquoi en est-il ainsi et quel rôle peut-il jouer?

\subsection{Approche méthodologique}

Cette étude a utilisé trois différentes approches méthodologiques de recherche qualitative pour collecter et analyser ces informations: les groupes de discussions dirigées (GDD), les entretiens individuels approfondis (EIA) et les ateliers d'autoanalyse (AAA). Toutes les données ont été recueillies au niveau de la communauté (hommes et femmes) et des prestataires communautaires (agents SBC, accoucheuses villageoises, tradipraticiens, agents de santé communautaire) en langue locale, le mooré.

\section{Site de l'étude}

L'étude était mise en œuvre dans les zones B et D du LSC. ${ }^{7}$ La zone B est la plus intense des deux zones expérimentales où les agents SBC travaillent actuellement, et où les formations sanitaires et leurs personnels ont été renforcés. La zone $D$ est une zone de comparaison où I'ONG "Save the Children" a récemment terminé un programme de soins de santé à base communautaire. L'étude a été menée dans la circonscription de l'un des centres de santé et la promotion sociale (CSPS) de chaque zone ; Komsilga pour la zone B et Rakaye pour la zone D.

\section{Collecte de données}

Etant donné la nature des données à collecter et du site de l'étude, il a été approprié d'utiliser des méthodes de recherche qualitative. Les membres de la communauté ont l'habitude de participer à des activités relatives aux questions de santé de la reproduction dans la région du fait de la présence du Laboratoire de Santé Communautaire. Cette étude a collecté des données auprès d'un certain nombre de groupes d'hommes et de femmes de la zone d'étude afin de mieux comprendre à partir d'une variété de perspectives les différents aspects des pratiques en matière de santé maternelle et infantile. Des groupes de jeunes célibataires ont été interviewés afin de comprendre leurs attentes quand ils auront leurs propres enfants, ainsi que leur rôle dans la famille en ce qui concerne les soins de santé. Des GDD ont été organisés avec des hommes et des femmes mariés ayant des enfants pour s'instruire de la façon dont ils prennent en charge les soins de santé, ainsi que de leurs expériences et de leurs attentes concernant leur partenaire. Les membres du

\footnotetext{
${ }^{7}$ Notons que le LSC regroupe 4 zones dont les interventions diffèrent d'une zone à l'autre. Zone $\mathrm{A}=$ renforcement des cliniques ; zone $\mathrm{B}=$ renforcement des cliniques et introduction des agents $\mathrm{SBC}$; zone $\mathrm{C}=$ zone témoins ; zone $\mathrm{D}$ = zone de comparaison (intervention de "Save the Children").
} 
comité de gestion de santé des CSPS, les agents SBC et les autres agents de santé ont été pris en compte dans l'échantillon de l'étude.

Deux équipes de quatre personnes chacune et composées d'un modérateur et de deux assistants ont exécuté chacune sept (7) groupes de discussions dirigées pendant une période de 12 jours. Les membres de l'équipe avaient déjà une expérience en recherche qualitative et en enquêtes dans le domaine de la santé. Avant la collecte des données proprement dite, les membres de l'équipe ont reçu une formation de cinq jours. Cette formation a inclus le pré-test des instruments de collecte des données. L'équipe a organisé un groupe de discussion, chaque matin, réservant les après-midi pour en faire le résumé et rédiger une synthèse sur les résultats. Les groupes de discussions ont été enregistrés sur magnétophone.

Pour ce qui concerne les ateliers d'auto-analyse, Mwangaza ${ }^{8}$ a supervisé, en collaboration avec les personnes ressources des équipes cadres du district, les deux ateliers d'auto-analyse avec les membres des comités de santé et le personnel du Ministère de la Santé. Les ateliers d'auto-analyse ont été enregistrés sur bande vidéo.

\section{Echantillonnage et population cibles}

Le tableau suivant décrit la population ayant fait l'objet de l'étude regroupée selon les instruments de collecte des données. La moitié du total des GDD, des interviews individuelles approfondies, des ateliers d'auto-analyse a été organisé dans chaque zone.

La sélection des groupes cibles a tenu compte des critères ci-dessus mentionnés mais aussi de la zone de couverture des formations sanitaires. Autour de la formation sanitaire, l'identification a distingué trois aires à savoir, les habitants qui sont à moins de cinq kilomètres de la FS, ceux qui sont entre 5 et 10 kilomètres et enfin les personnes résidant à plus de 10 kilomètres de la FS. Cette façon de procéder est soutenue par le postulat que le comportement, les connaissances et les perceptions sont influencés par la proximité à une formation sanitaire. Les responsables des formations sanitaires et les délégués des villages situés dans ces aires ont aidé à la sélection des groupes cibles. Chaque GDD a réuni 8 personnes au minimum et 12 personnes au maximum. En moyenne, le nombre de participants était de 10 personnes.

8 . Mwangaza-Action est une ONG locale affiliée à la Ligue Coopérative des Etats-Unis d'Amérique (CLUSA), spécialisée dans les questions de mobilisation et de participation communautaire en générale et avec la population du Bazèga en particulier. 


\begin{tabular}{|l|c|c|c|c|}
\cline { 2 - 5 } \multicolumn{1}{c|}{} & \multicolumn{2}{c|}{ KOMSILGA } & \multicolumn{2}{c|}{ RAKAYE } \\
\cline { 2 - 5 } \multicolumn{1}{c|}{} & Hommes & Femmes & Hommes & Femmes \\
\hline GDD adolescents de 15 à 19 ans & 1 & 1 & 1 & 1 \\
\hline $\begin{array}{l}\text { GDD personnes mariées de } 20 \text { à } 29 \\
\text { ans ayant un enfant < 5 ans }\end{array}$ & 1 & 1 & 1 & 1 \\
\hline $\begin{array}{l}\text { GDD avec personnes mariées de 30 à } \\
49 \text { ans ayant un enfant < 5 ans }\end{array}$ & 1 & 1 & 1 & 1 \\
\hline $\begin{array}{l}\text { GDD avec personnes de plus de 49 } \\
\text { ans ou "yaaba" (grand-mères) }\end{array}$ & & 1 & & 1 \\
\hline EIA agents SBC & 1 & 1 & 1 & 1 \\
\hline EIA agents de santé communautaire & 1 & 1 & 1 & 1 \\
\hline EIA accoucheuses villageoises & 1 & 1 & 1 & 1 \\
\hline EIA tradithérapeutes & 1 & 1 & 1 & 1 \\
\hline
\end{tabular}

\section{Analyse des données et rédaction du rapport}

Sur le terrain, à la fin de chaque journée de travail, une réunion de synthèse était organisée afin de revoir les prises de notes et les problèmes d'ordre méthodologiques. Chaque interview et chaque GDD faisait l'objet d'un rapport amendé par le groupe.

Après la collecte entière des données, il a été organisé un atelier de quatre jours afin de procéder à une analyse primaire du contenu des GDD et des entretiens individuels approfondis. Cet atelier a regroupé l'équipe de recherche, des représentants de la DSF. II s'agissait d'impliquer les enquêteurs dans le processus d'analyse afin de permettre une meilleure interprétation des données. L'analyse des données et la rédaction du rapport ont bénéficié de l'appui technique du Population Council. 


\section{RESULTATS}

\subsection{Maternité sans risque (MSR)}

\subsubsection{Connaissances, perception et comportement}

\section{Pendant la grossesse}

\section{Les signes de reconnaissance d'une femme en grossesse et annonce de la grossesse à l'entourage}

La question est de savoir comment reconnaît-on qu'une femme est enceinte au sein de la communauté où l'étude a été réalisée. De la réponse à cette question, on peut retenir les éléments synthétiques suivants :

D'abord les signes physiques: le blanchissement du visage et des mains ("elle devient comme un bébé", homme marié de 20 à 29 ans, Rakaye), l'apparition de boutons sur le visage, le grossissement des seins et les œdèmes des pieds, le grossissement ou amaigrissement de la femme sont autant de signes répertoriés qui indiquent qu'une femme est enceinte. Cependant pour tous les groupes, l'on reconnaît qu'une femme est enceinte que lorsque la grossesse est visible, c'est-àdire autour du troisième ou quatrième mois . Par contre, pour les adolescentes, c'est seulement au bout du septième mois qu'elles reconnaissent l'état de grossesse d'une femme.

Le deuxième type de signes évoquant une grossesse comprend l'arrêt de certaines activités physiques comme piler le mil, cultiver, puiser de l'eau. En dehors des activités physiques, l'abandon de pratiques religieuses a été mentionné ; ainsi, la femme musulmane qui arrête d'effectuer ses prières quotidiennes est considérée comme étant enceinte.

Le troisième type de signe est constitué des signes sympathiques de la grossesse : on reconnaît qu'une femme est enceinte lorsqu'elle sélectionne ses aliments, vomit (en début de grossesse), dort et crache beaucoup.

A qui et comment une femme annonce-t-elle qu'elle est enceinte ? C'est très souvent au mari qu'est annoncé en premier la grossesse "parce qu'il en est responsable." L'annonce au mari en premier a pour souci de le responsabiliser quant aux charges subséquentes ou pour qu'il reconnaisse la paternité de la grossesse ou

La grossesse s'annonce en priorité :

- au mari

- à la belle-mère

- à la mère

- à une meilleure amie co-épouse encore pour une gestion de leurs rapports sexuels à l'avenir. L'annonce au mari se fait généralement sur le lit conjugal ou par l'intermédiaire des "vieilles," belles-mères notamment. Le recours aux vieilles femmes est lié au fait qu'elles ont acquis une grande expérience en la matière. Enfin, une femme peut annoncer à sa co-épouse ou à son entourage qu'elle est enceinte à condition qu'il y ait de l'entente entre les deux parties. Cela est lié au fait que l'on pense qu'autrui peut vous jeter un mauvais sort qui serait à l'origine de complications pendant la grossesse ou au cours de l'accouchement. 
Les propos utilisés par la femme pour annoncer sa grossesse sont codifiés et reconnus comme tels par la communauté. La femme dira à son mari : "je n'ai pas vu mes règles" ou encore "j'ai attendu mes règles en vain, je crois que ce doit être une grossesse" ou "j'ai changé, je ne suis pas comme avant". Le déménagement de la femme dans la case de sa belle-mère ou le sevrage de l'enfant encore porté au dos sont autant de formes d'expression utilisées par la femme enceinte pour annoncer sa grossesse.

Avec ce nouvel état, la femme enceinte entame une nouvelle étape de sa vie, avec des interdits et des recommandations concernant son alimentation, ses comportements sociaux et sexuels.

\section{Les recommandations et interdits alimentaires pour une femme enceinte}

Tout au long de la grossesse, la femme se voit contrainte de suivre des recommandations et des interdits d'ordre alimentaire, thérapeutique et social, voire sexuel.

\section{Sur le plan alimentaire}

Des interdits alimentaires sont prescrits à la femme enceinte dans le but d'éviter les complications ultérieures. C'est ainsi qu'à la femme enceinte, il est recommandé d'éviter de trop manger les aliments salés et sucrés, de ne pas boire du lait, ce qui, selon les enquêtés, permet d'éviter d'avoir un gros bébé qui est source de complications à l'accouchement. II lui est également interdit de consommer du miel car un aliment auquel la communauté attribue un pouvoir abortif. Les excitants tels que l'alcool ("la consommation d'alcool devrait être réduite d'un quart en cas de grossesse," groupe de femmes de lus de 40 ans), le tabac et le café sont des aliments interdits aux femmes enceintes selon la communauté.

A côté des interdits alimentaires prônés par la minorité dans les deux localités de l'étude, on retiendra de la majorité que la femme enceinte devrait bien manger "parce qu'elle mange pour deux," elle devra consommer de la viande, du poisson, des œufs, du riz, du tô (pâte de maïs ou de mil s'accompagnant d'une sauce gombo ou feuilles de baobab ou de courgettes), du baabenda (sauce de feuille de haricot ou d'oseille et de céréale) sauf en cas d'intolérance ou de malaise de la femme. En d'autres termes, il n'y a pas de restriction alimentaire, "la femme doit manger tout ce que son ventre réclame pour avoir la bonne santé." Les prestataires communautaires ont classé les aliments selon leurs apports à l'organisme de la femme enceinte (encadré ci-dessus). Ils tiennent ces
Les aliments conseillés pour une femme enceinte

Laafi riibo (aliments donnant la santé)

- Feuilles d'oseille

- Feuille de haricot

- Feuille de courgette

- Feuilles de baobab.

Ziim dibo (aliments donnant du sang)

- Poulet

- OEufs

- Poisson.

Pang riibo (aliments donnant la force)

- Mil

- Maïs.

Rii noondo (mets succulents) selon les adolescentes seulement:

- $\quad$ Tô (pâte de farine de mil ou de maîs)

- $\quad$ Soupe de pintade potassée

- Lait. 
informations des prestataires cliniques qui les leur ont enseigné pendant leur formation.

\section{Sur le plan thérapeutique}

En matière de thérapie, les différents groupes estiment que le traitement moderne est le meilleur. Par conséquent, ils recommandent en priorité, le traitement avec des produits prescrits à la formation sanitaire. Cependant la pilule est considérée comme un produit abortif, "la femme enceinte doit éviter de prendre des pilules car cela peut provoquer un avortement."

Le traitement traditionnel est controversé; pour les femmes de plus de 40 ans ce type de traitement est le recours efficace en cas d'avortements répétés. Devant de tels cas, elles réfèrent systématiquement les patientes aux tradipraticiens. Cependant, il est déconseillé de se référer aux tradipraticiens "parce que les produits traditionnels peuvent provoquer un avortement." Le groupe des adolescents font entièrement confiance au traitement traditionnel qui, selon eux, contribue à "éloigner les mauvais esprits."

\section{Sur le plan des relations sexuelles}

Pour ce qui concerne les relations sexuelles pendant la grossesse, deux tendances se dégagent :

Une première tendance majoritaire des hommes et des femmes estime qu'il est interdit de poursuivre les relations sexuelles avec une femme enceinte après le $3^{\text {ème }}$ voire le $4^{\text {ème }}$ mois, car "ce qui a permis de concevoir l'enfant ne peut lui faire du mal." Ou encore, "ce qui a amené l'enfant ne peut pas le gâter" (femme de 30 à 39 ans). Selon cette tendance, passée la période sus mentionnée "la poursuite des activités sexuelles avec une femme enceinte fait qu'à l'accouchement l'enfant est couvert de substance huileuse et gluante ou de saletés" (rappelant le sperme).

La seconde tendance minoritaire pense qu'il est permis d'avoir des relations sexuelles avec une femme enceinte jusqu'à l'approche de l'accouchement.

\section{Autres interdits et recommandations d'ordre social}

Des autres recommandations d'ordre social, on peut retenir, la réduction des grands efforts physiques (coupe du bois, cultures, port de lourdes charges, transport de l'eau, la longue marche...), la position assise avec jambes fléchies, le repos excessif, enfin, il faut éviter de s'approcher d'une source de chaleur (foyer de la cuisine). Tous ces éléments sus mentionnés pourraient être à l'origine d'un accouchement prématuré. 


\section{Les signes et causes de complications au cours de la grossesse}

\section{Les causes de complications}

La grossesse de l'adolescente est perçue comme une grossesse à risque. Les communautés interviewées l'ont mentionné en parlant de "grossesse de la fille non mûre;" cependant la question fondamentale est de savoir quand est-ce que la fille est dite "mûre" quand on sait que la plupart des filles de la zone d'enquête se marie autour de 17 ans, c'est à dire pendant l'adolescence.

La femme enceinte devrait continuer à travailler à minima, si non elle peut s'exposer à des complications; elle devrait par conséquent éviter "l'inactivité prolongée."

L'infidélité est perçue comme cause de complication au cours de la grossesse. Toute infidélité est récompensée par une punition des ancêtres et de ce fait, si le fruit de cette infidélité est une grossesse, celle-ci connaîtra inévitablement des complications.

Principaux éléments laissant présager une complication au cours de la grossesse :

Douleurs au bas-ventre et au ventre Etroitesse du bassin de la femme Taille de la femme (petite)

Vomissement et enflement des pieds.

\section{Les signes de complications}

Plusieurs symptômes ont été mentionnés comme évocateurs de complications au cours de la grossesse : les douleurs au bas-ventre accompagnées de saignement ou "poudgré," les douleurs au ventre, "lorsque le bébé bouge beaucoup dans le ventre de la mère," les vomissements et l'œdème des membres inférieurs, la fièvre. Pour les cas de complications ou en dehors, quel est l'itinéraire thérapeutique des femmes pendant la grossesse?

\section{L'itinéraire thérapeutique}

Pour les conseils, les femmes se réfèrent généralement à la formation sanitaire (FS) mais cela varie en fonction des zones et des groupes.

Si à Komsilga le recours premier est la FS, à Rakaye, la priorité est accordée à la belle-mère ou la "vieille femme" compte tenu de son expérience et de l'exigence de la tradition. Selon les adolescents, les agents professionnels de santé "peuvent détecter les anomalies du fœtus et donner les conseils nécessaires." La plupart des groupes de femmes prétendent aller à la FS en priorité, puis vers les agents SBC femmes et les accoucheuses villageoises tandis qu'une minorité de femmes mariées (20 à 29 ans) ont recours aux conseils des tradipraticiens surtout lorsqu'elles ne sont pas satisfaites des conseils des agents SBC. La préférence va à l'accoucheuse villageoise plus proche et souvent expérimentée.

Ordre de priorité pour les recours en cas de besoin de conseils

- Formation sanitaire

- Agent SBC

- Accoucheuse villageoise

Ordre de priorité pour les recours

En matière de soins, les jeunes femmes (20 à 29 en cas de besoin de soins ans) citent en premier lieu la FS, puis viennent - Formation sanitaire les accoucheuses villageoises. Pour les femmes - Accoucheuse villageoise d'âge plus mûr, la préférence va à la FS car - Agent SBC disent-elles : "notre espoir de vivre est dans leurs 
mains." Les agents de SBC jouissent de la confiance des différents groupes pour ce qui concernent les conseils en matière de survie de l'enfant et des problèmes liés à la grossesse, sauf pour celui des hommes mariés (20 à 29 ans) qui les considèrent comme ayant "une connaissance limitée." Pour les hommes mariés (30 à 49 ans), il faut s'y référer car les maladies qui prévalent sont dites modernes et par conséquent les soins aussi le sont: "les maladies d'aujourd'hui sont des maladies de blancs." Ce constat constitue une opportunité positive qu'il faut exploiter si l'on veut introduire des activités de survie de l'enfant et maternité sans risque dans le paquet de services offerts par les agents de services à base communautaire ; ces activités ne font pas encore partie intégrante des responsabilités des agents.

\section{Les rites et coutumes à l'endroit de la femme enceinte}

Dans les communautés traditionnelles, il y a des rites et coutumes qui sont appliqués à la femme en grossesse. Etant donné la proximité de la zone d'étude à la capitale du pays et les phénomènes de migration, l'on penserait que de telles pratiques tendent à s'amenuiser. Au cours de cette étude, il s'avère que beaucoup de ces pratiques persistent malgré la proximité de la ville de Ouagadougou et les campagnes de sensibilisation. La connaissance des rites et coutumes est variable en fonction des groupes. Les adolescents ne connaissent que très peu de choses en la matière ; la seule chose qui a été mentionnée est l'aumône qui est souvent fait afin que la femme puisse accoucher sans difficulté. Cependant, les autres groupes parlent de rites et coutumes jadis pratiqués mais peu répandus de nos jours.

La pratique coutumière la plus souvent évoquée est le "sêgne puga:" à la suite d'une cérémonie rituelle, on attache un fil autour de la ceinture de la femme ou en bandoulière afin que l'enfant qui va naître soit reconnu comme un membre "authentique" de la communauté. Cette pratique est surtout appliquée aux jeunes filles offertes en mariage et qui sont enceintes pour la première fois, dans le but de les empêcher de fuir le domicile conjugal et de légitimer la grossesse et faciliter l'accouchement du premier enfant. ${ }^{9}$ Le second rite consiste à ce que la mère de la femme enceinte aille chez son beau-fils pour lui préparer un repas et par la même occasion lui annoncer la nouvelle de la grossesse. $\mathrm{Ce}$ rite est célébré environ 3 mois avant l'accouchement. Enfin, pour la majorité des groupes enquêtés, lorsqu'une femme est enceinte, on consulte le devin (baga) pour connaître d'avance le déroulement de l'accouchement et partant prévenir les complications éventuelles; cela revient aux "vieux" et des sacrifices sont faits à cet effet.

Principaux signes précurseurs de complication pendant

l'accouchement:

- Mauvaise présentation du bébé (dos, fesses, main)

- Durée du travail prolongé (roog toogo),

- Petite taille de femme et du bassin,

- Rétention placentaire.

Les causes de complication sont:

- Disputes pendant la grossesse,

- Adultère

\footnotetext{
9 Pour cela, les vieilles femmes se regroupent, font moudre le mil, préparent du "dolo" (boisson locale alcoolisée à base de mil rouge). Elles en boivent et font boire à la femme enceinte en faisant semblant de la battre. Quant à la description détaillée d'un tel rite, c'est dit-on "un secret de femmes."
} 


\section{Au cours de l'accouchement}

\section{Les signes de complication}

Quels sont les signes précurseurs de complications tels que perçu par la communauté? Les groupes des adolescents et des femmes de 20 à 29 ans ignorent l'existence de signes précurseurs de complications pouvant survenir au cours de l'accouchement ; selon eux, seul l'infirmier ou encore les "vieilles femmes" peuvent le savoir. Cependant, pour l'ensemble des groupes réunis pour les GDD, il revient souvent que les causes et signes suivants peuvent présager un accouchement compliqué :

- "Lorsqu'au cours du travail, la femme n'arrive pas à expulser le bébé malgré ses efforts."

- "Si au cours de sa grossesse la femme a eu des disputes avec quelqu'un, il s'en suit une malédiction rendant difficile l'accouchement, surtout, s'il s'agit du mari."

- "Lorsque le mari n'est pas le géniteur de la grossesse (cas d'adultère)."

\section{L'itinéraire thérapeutique}

L'étude s'est intéressée à explorer l'itinéraire thérapeutique de la communauté des femmes enceintes et en matière de soin de l'enfant.

\section{Conseils}

Ainsi, pour ce qui concerne les conseils, les enquêtés à l'unanimité pensent que les femmes doivent se référer à la formation sanitaire où les agents de santé sont formés à cet effet : "Ils peuvent même donner l'heure de l'accouchement ou référer à une formation mieux adaptée en cas de complications." Les accoucheuses villageoises sont aussi des prestataires fréquentées parce qu'elles sont formées et sont proches des populations: "ce sont des mécaniciennes de l'accouchement" (hommes de 30 à 49 ans). Dans tous les cas, les enquêtés des différents groupes marquent leur préférence pour la formation sanitaire pour les raisons contenues dans l'encadré cicontre. Cependant, le recours à la formation sanitaire pour les conseils n'est pas

Raisons évoquée pour recourir à la formation sanitaire pour des conseils ou des soins

- Sécurité : "il n'y a pas de risque d'infection par le tétanos,"

- Qualité des soins, Meilleur suivi post-partum. systématique; les femmes s'y réfèrent en cas de maladie ou de complications.

\section{Soins}

De même que la FS n'est pas de fréquent recours pour les conseils, l'on note le même cas de figure pour ce qui concerne les soins, sauf en cas de complications apparentes comme le travail anormalement long; cependant au cas où la prolongation du travail est liée à des propos injurieux tenus à l'endroit de son mari, il est préconisé d'aller voir un tradithérapeute chez qui la femme devrait se gargariser la bouche et cracher "puuss nooré" afin de trouver la délivrance.

Les adolescentes pour la plupart ne savent pas la conduite à tenir en cas d'apparition de signes de complication au cours de l'accouchement. Selon eux, seuls 
le personnel de santé est à même de déceler ces signes. Ils ajoutent qu'en cas de grossesse adultérine, la femme se doit de faire des aveux à son mari avant de pouvoir accoucher, si non l'accouchement sera inévitablement sujet à une complication.

\section{Les rites, coutumes et religions}

Le traitement moderne est souvent allié au traitement traditionnel. En effet, des rites et coutumes sont souvent pratiqués en l'endroit de la femme au moment de l'accouchement. Pour les femmes de plus de 49 ans l'aumône de mil et de pièces d'argent offert aux marabouts et autres mendiants peut faciliter l'accouchement.

Parfois certains rites sont faits à titre préventif: pour connaître l'issue de la grossesse, un sacrifice de poulet est fait: on l'égorge et on le jette, si le poulet tombe sur le dos, cela signifie que les ancêtres ont accepté l'offrande (hommes de 20 à 29 ans et de 30 à 49 ans), ce qui signifie que la femme accouchera sans problème.

Pour les adolescentes, seul un rite issu de la religion islamique est connu; il s'agit de boire du "nassa" qui est un liquide obtenu après le lavage des écritures islamiques écrites sur un support en bois "walga." Un autre rite musulman connu est le "doua" qui consiste en l'aumône de galettes ou de sésame offerte selon les prescriptions du marabout.

\section{La période du post-partum}

\section{Les signes de complication}

Les signes les plus mentionnés comme précurseurs des complications à cette phase de la maternité sont l'hémorragie, les maux de ventre, les vertiges, et les douleurs pelviennes. Ces signes surviennent surtout au cours du post-partum immédiat, c'està-dire avant le quarantième jour après l'accouchement. Au-delà des 40 jours, aucun signe de complication n'est pratiquement mentionné.

\section{L'itinéraire thérapeutique}

\section{Conseils}

Dans la période suivant l'accouchement, la plupart des enquêtés désignent la formation sanitaire comme la structure de recours pour les soins. Mieux, ils y portent leur préférence d'une part à cause de l'expertise et l'expérience du personnel et la disponibilité des médicaments. Cependant, une minorité des femmes de 30 à 49 ans, pense que la femme accouchée peut se référer aux agents de services à base communautaire et aux tradipraticiens (Rakaye).

\section{Soins}

La prise en charge des complications ou des signes de complications dans le postpartum fait appel au traitement traditionnel. II s'agit de décoction de racine que la femme boit et utilise pour ses toilettes. 


\section{Rites et coutumes}

Contrairement à la période de la grossesse et de l'accouchement où certaines croyances restent fortes et persistantes, la période du post-partum est sujette à très peu de rites et de coutumes. Pour la plupart des enquêtés, tous âges confondus, ils ne connaissent pas de rites et coutumes concernant la femme dans les suites de l'accouchement. Cependant, chez les musulmans le baptême se fait le septième jour et à partir de ce moment, la femme est autorisée à sortir tandis que chez les animistes, la femme peut sortir à partir du quatrième jour suivant l'accouchement.

\section{Les recommandations et interdits alimentaires pour une femme nouvellement accouchée}

Contrairement à la période de la grossesse, la période du post-partum est soumise à très peu d'interdits.

\section{Sur le plan alimentaire}

D'un point de vue alimentaire, l'accouchée récente (post-partum immédiat) ne doit pas manger des aliments froids ou épicés, les femmes âgées de 30 à 49 ans déclarent qu'elle ne doit pas manger des pois de terre ou des haricots ou tout aliment à base de ces deux aliments à cause des risques de ballonnements préjudiciables à la mère et à l'enfant avec risque de diarrhée ou de maux de ventre de l'enfant. Le repas d'une nouvelle accouchée doit être léger, surtout lors du postpartum immédiat, chaud ce qui permet de maintenir "le ventre de l'accouchée au chaud" et ce qui favoriserait "la guérison de la plaie provoquée au niveau de l'utérus par l'accouchement."

Dans le post-partum lointain, l'accouchée peut reprendre ses habitudes alimentaires voire manger tout ce dont elle a envie pour récupérer ses forces. Elle devrait en particulier basé son régime sur le "laafi ribo" (aliment donnant la santé), le "pang ribo" (aliment donnant la force) et le "zim ribo" (aliment donnant du sang).

\section{Sur le plan thérapeutique}

Du point de vue de la thérapeutique, les enquêtés en général, ne connaissent pas d'interdits ni dans le post-partum immédiat ni dans le post-partum lointain. Ils recommandent dans l'ensemble de se référer aux FS. Selon la majorité des enquêtées, les nouvelles accouchées devrait se rendre dans les FS en vue de bénéficier des services de planification familiale, de vaccination aussi bien pour la mère que pour l'enfant. Cette attitude positive serait peut être liée à la présence des agents SBC.

\section{Sur le plan sexuel}

L'unanimité des enquêtés déconseillent les relations sexuelles dans le post-partum immédiat car "la plaie n'est pas encore guérie" et par ailleurs, l'enfant peut tomber malade surtout s'il est essentiellement nourri au sein. Pour la période du post-partum lointain, les avis sont divergents; tandis que certains enquêtés estiment que la femme peut reprendre progressivement les rapports sexuels à la demande du conjoint afin d'éviter d'éventuelles disputes, les adolescentes pensent que les femmes ne devraient pas avoir des rapports sexuels avant plusieurs années deux à trois ans après un accouchement. Les enquêtés pensent que lorsqu'un enfant est au 
sein, il faut éviter les rapports sexuels avec sa mère car le sperme passe dans le lait et cela entraîne de la diarrhée chez l'enfant.

\subsubsection{Implication des hommes}

II ressort que les rôles reconnus appropriés ou exigés des maris pendant la grossesse de leur épouse ne sont pas toujours exécutés par ceux-ci. Les enquêtés suggèrent que des actions soient prises pour impliquer les hommes pendant les grossesses de leurs épouses. Plus précisément, d'autres ont recommandé que des séances de sensibilisation soient menées auprès des hommes des différentes communautés afin qu'ils aient une même vision des choses et qu'ils s'unissent pour entreprendre des actions entrant dans le cadre de l'amélioration de la santé de tous. Un tradipraticien de Rakaye partage cet avis car selon lui : "si tu as quelqu'un pour te conseiller, t'éclairer l'esprit, si tu te lèves, tu vois clair."

Le rôle des hommes tel que vécu actuellement est certainement lié au phénomène social et culturel qui leur confère ce rôle ; en d'autres termes, ce sont des rôles liés au genre. Ainsi, un agent SBC (homme) a remarqué que l'éducation des hommes et leur implication dans le processus et le suivi de la grossesse pourrait être salutaire s'ils permettent de mieux connaître et accepter la PF. Pour lui, les grossesses rapprochées constituent l'une des principales causes de complications et il suggère pour cela que: "les femmes s'entendent avec leur mari afin que leurs grossesses soient vraiment désirées. En ce moment, il leur est plus facile de bien les mener à terme." Cependant, la plupart des groupes et des interviewés estiment que le rôle des hommes est important.

\section{Sur le plan alimentaire}

Les hommes apporteraient des aliments nourrissants comme la viande, le poisson, les œufs et certaines céréales aux femmes enceintes et accouchées. Certains ont affirmé que l'homme devrait faire un effort pour doter sa femme de ce dont elle a besoin comme aliments ou ce dont elle a envie, si celle-ci est enceinte ou accouchée.

\section{Sur le plan du travail}

Les hommes devraient aider les femmes enceintes dans les travaux de force tels que puiser l'eau, chercher et casser du bois, faire la lessive, moudre les céréales. En d'autres termes, les femmes souhaiteraient avoir le soutien des hommes dans les travaux qui socialement et culturellement leur sont dévolus surtout si elles sont enceintes.

\section{Sur le plan de la prise en charge de la grossesse}

Un des aspects positifs de l'implication des hommes est qu'ils accompagnent toujours la femme à la formation sanitaire quand il s'agit d'accouchement. A ce propos, un membre de la communauté (hommes de 30 à 49 ans) de Komsilga dit ceci : "si je pouvais rentrer dans la salle, j'allais le faire pour pleurer un peu avec ma femme." Ce n'est pas le cas quand il s'agit des consultations prénatales. Aussi, les femmes souhaiteraient que les hommes les accompagnent de temps à autres à la formation sanitaire durant la grossesse. 
Le rôle de l'homme se voit aussi dans la prise en charge des produits nécessaires pour faciliter la grossesse, l'accouchement et le post-partum; il s'agit essentiellement des produits pharmaceutiques prescrits à la femme ainsi que le matériel requis pour l'accouchement (gants, antiseptique, coton,...). Tant les femmes et les hommes estiment que des activités génératrices de revenu (commerce de bétail par exemple) pourraient aider les hommes à être mieux outillés sur le plan financier pour la prise en charge des frais occasionnés durant la grossesse et l'accouchement.

En plus des rôles ci-dessus mentionnés, les enquêtés hommes et femmes, mais surtout les femmes, ont souhaité voir le rôle de l'homme pendant la grossesse, l'accouchement et la période post-partum s'élargir à d'autres aspects :

\section{Sur le plan de la communication}

Les femmes souhaiteraient que les hommes puissent communiquer avec les agents de santé à propos des conditions de leur femme, c'est-à-dire précisément de discuter avec le personnel afin d'avoir des conseils à propos du déroulement de la grossesse.

\section{Sur le plan moral}

Un homme a le devoir d'apporter un soutien moral à sa femme pendant les périodes de grossesse et après l'accouchement; l'homme devrait éviter de faire de la peine aux femmes pendant cette période car disent les enquêtés, "on a remarqué que les femmes peuvent être plus émotives et irritables lorsqu'elles sont enceintes." La traditionnelle crainte de frustration et de désaccord pendant la grossesse qui pourrait être source de complications à l'accouchement explique peut-être pourquoi quelques enquêtés trouvent particulièrement importants de maintenir une bonne entente avec la femme.

Enfin l'une des tendances est que l'homme n'a pas de rôle particulier à jouer au moment de l'accouchement excepté le fait qu'il doit "attendre dehors dans l'angoisse" (femmes de 30 à 49 ans).

II apparaît une différence entre les groupes consultés que les GDD de Rakaye étaient moins enclins à proposer des rôles pour les hommes que ceux de Komsilga. Cela serait sans doute lié à l'effet de la religion musulmane prédominante à Rakaye. En outre, les groupes de femmes plus âgés (30 à 49 ans) ont peut-être beaucoup plus mis l'accent sur le rôle traditionnel des hommes (consultations chez les devins, place de choix pendant l'acte de procréation).

\subsubsection{Implication des agents SBC dans les prestations de services de maternité sans risque et de survie de l'enfant}

Un des volets de l'étude a été de recueillir les avis de la communauté dans laquelle évoluent les agents SBC quant à leur éventuelle implication à la promotion de la maternité sans risque ainsi que la survie de l'enfant. Les avis exprimés concernaient l'acceptabilité de ces SBC dans ce domaine et l'approche genre (préférence dans le sexe : homme ou femme pour mener de telles activités). 


\section{Acceptabilité des agents $S B C$}

De façon unanime, tous les différents groupes cibles interrogés pendant l'enquête sont d'accord pour que les agents SBC mènent des activités entrant dans le cadre de la maternité sans risque et de la survie de l'enfant (MSR/SE)

Les principales raisons de cette acceptabilité se répertorient dans l'encadré ci-dessus. II apparaît donc que les agents SBC sont acceptés pour mener des activités en matière de MSR/SE du fait de leur proximité avec les utilisateurs, du fait qu'il n'y a pas un long interrogatoire comme pendant une visite dans une formation sanitaire. De plus, la communauté voit à travers les activités liées à la PF déjà menées par les agents $\mathrm{SBC}$, une opportunité pour y intégrer des activités complémentaires en matière de MSR/SE.

\section{Approche par le genre}

L'une des questions à laquelle, l'étude a souhaité répondre était aussi de savoir si le sexe de l'agent SBC était déterminant si celui devait effectivement mener des activités en matière de MSR/SE. Les avis divergent d'un groupe cible à un autre et à l'intérieur d'un même groupe. La majorité des enquêtés ne voient pas d'inconvénients à ce qu'un agent SBC de sexe masculin ou féminin soit formé pour offrir des services ou conseils et de prendre en charge les femmes enceintes dans leur communauté. La majorité des groupes de femmes mariées âgées de 20 à 29 ans, d'hommes mariés de 30 à 49 ans et de femmes de plus de 49 ans n'ont pas de préférence pour le sexe. Elles pensent également que l'agent SBC doit s'entretenir avec le couple en même temps au lieu de le faire séparément. Ainsi, ils auront toutes les informations qu'ils désirent en même temps; de plus, le couple peut continuer la discussion après le départ de l'agent SBC.

Une tendance particulière notée au niveau des groupes des adolescents est que, pour une grande confidentialité et de sérieux, "les agents SBC hommes doivent s'adresser aux femmes pour les causeries." lls justifient cette position par le fait que cette distinction permet d'éviter la gêne ou la honte, (surtout pour les femmes qui ont honte de s'adresser aux hommes), et facilite la communication : "chaque personne a son petit secret qu'elle ne veut pas dire au sexe opposé" (groupes d'adolescents de Rakaye et de Komsilga).

\subsection{Survie de l'enfant (SE)}

\subsubsection{Soins de l'enfant}

Où se réfèrent les femmes pour les soins des enfants? La plupart des enquêtés pensent que les femmes accouchées peuvent se . référer à la formation sanitaire • parce que cela est plus
"La section du cordon ombilical se fait à l'aide d'instruments propres"

- $\quad$ "On prescrit des médicaments nécessaires relativement à bon prix"

"Les prestataires sont qualifiés en la matière"

"Il y a des soins préventifs tels que la vaccination (polio, BCG) à partir du 35ème jour de la naissance”. 
sécurisant. Les raisons de cette perception sont contenues dans l'encadré ci-contre.

Cependant, la majorité des populations cibles enquêtées estiment également que l'on peut aller voir les tradipraticiens ou les "vieilles" pour le cas de maladies infantiles telles que:

- Le "saimomdé," (anus rouge dont l'interprétation dans le jargon médical est assimilée à l'éryrhème fessier du nourrisson)

- Le "zoulongo" (qui signifie enfoncement de la fontanelle antérieure de l'enfant).

La collecte des données auprès des tradipraticiens montrent qu'ils donnent des prestations de services portant sur la survie de l'enfant ; le contenu de leurs conseils aux mères couvrent les aspects suivants :

- Bien faire la toilette du nourrisson et de l'enfant afin qu'ils soient en bonne forme.

- Amener l'enfant à la FS le $35^{\text {ème }}$ jour après l'accouchement pour les vaccins et selon les rendez-vous donnés par l'agent de santé.

- Gaver et purger le nourrisson avec une décoction à base de plantes. Ils ajoutent que l'on ne doit pas le faire avec n'importe laquelle décoction car cela pourrait entraîner des maladies.

Une précision a été donnée pour ce qui concerne l'administration des décoctions: jusqu'à l'âge de 3 à 4 mois, c'est le "yaryamdé" et le "zankoné" qui sont donnés au bébé pour le fortifier. A partir du $5^{\text {ème }}$ mois, on donne le "sogloumdaga." A partir d'un an, on conseille de purger l'enfant avec des décoctions aigres.

En conclusion, les tradipraticiens allient une prise en charge moderne et traditionnelle. Une intervention devraient prendre en compte ce public cible. C'est selon eux, un enfant en bas âge peut vomir régulièrement ou ne grandir soit parce que le lait tété est trop sucré ou amer et ce traitement ne peut être que traditionnel. ${ }^{10}$

\subsubsection{Nutrition de l'enfant}

L'idée maîtresse qui revient le plus souvent est que "I'homme doit prendre en charge l'alimentation de la mère et de l'enfant." Si la femme mange bien, elle nourrira mieux son enfant. Pour cela la tâche essentielle de l'homme est de cultiver la terre et d'acheter du sucre. Pour les hommes et les femmes mariés, il faut offrir au bébé un régime alimentaire spécial riche en vitamines (oranges, foie, mangues...) lorsque celui-ci commence à manger.

Le menu familial devrait être amélioré, selon les adolescentes et les femmes de plus de 49 ans avec un apport en poisson, viande ou œufs au profit de la nouvelle mère pour que celle-ci puisse avoir suffisamment de lait pour l'enfant. Les adolescents

\footnotetext{
${ }^{10}$ Pour un bébé qui vomit régulièrement ou qui ne grandit pas, la cause se trouve dans la qualité du lait de la mère. Le lait peut-être d'un goût amer (bistoogo) ou sucré (biissido). Ils expliquent que pour reconnaître le "biistoogo," on trait le lait de la femme, ensuite on le met dans une coke de noix de karité, puis on y ajoute une fourmi. Si la fourmi meurt, cela signifie que le lait est amer. Quant au lait sucré, on le reconnaît par le fait que l'enfant vomit régulièrement. Les tradipraticiens ajoutent que pour remédier à ces cas de figures et permettre à l'enfant d'avoir du "bon lait," la mère doit aller en brousse très tôt le matin et attraper ou tuer un oiseau appelé "kiiga." Ensuite, elle arrache des feuilles de karité en demandant du bon lait. Ainsi, elle laisse le "mauvais lait" à l'arbre et retourne à la maison avec du bon lait.
} 
préconisent l'achat de biberon et de Cérélac (céréale industrielle) au cas où la mère manquerait de lait.

\subsubsection{Rites et coutumes relatifs à la survie de l'enfant}

Pour ces volets rites et coutumes, plusieurs cas ont été identifiés dans les deux zones d'étude.

\section{Selon les animistes}

- "Rooga yiibou"' (sortie officielle de la nouvelle accouchée) qui survient le $3^{\text {ème }}$ jour après la naissance du bébé si celui-ci est de sexe masculin ou le $4^{\text {ème }}$ jour s'il s'agit d'une fille. La célébration de cette coutume est destinée à la présentation officielle de l'enfant à la communauté. A cet effet, on prépare du "tô" pour les enfants de la cour et un poulet pour les vieilles femmes. Une cuisse de ce poulet est offerte à l'accoucheuse principale et les ailes aux autres assistantes. A cette même circonstance, on rase la tête du nouveau-né. La procédure d'identification du nom du nouveau-né est une partie non moins importante car elle préside aux destinées de sa survie.

\section{Selon les musulmans}

- C'est "zouloukri" (baptême) célébré le $7^{\text {ème }}$ jour après la naissance du bébé. Ce jour-là, on rase la tête de l'enfant pour enlever les cheveux dits "sales" et on lui choisit un prénom selon le calendrier musulman ou au désir des parents. A l'occasion, on tue un mouton et des poulets que l'on prépare avec du riz et des boissons sucrées. Cette cérémonie est dirigée par un groupe de musulmans. Des cadeaux sont offerts au nouveau-né (savon, vêtements, argent). Des réjouissances populaires peuvent être organisées la veille. 


\section{RESULTATS DES ATELIERS D'AUTO-ANALYSE}

L'objectif de ces ateliers d'auto-analyse (AAA) était d'appuyer les représentants villageois et les prestataires communautaires à identifier des propositions d'intervention qui permettront de résoudre les problèmes liés à la maternité sans risque et à la survie de l'enfant dans les deux zones du LSC.

De façon spécifique les ateliers d'auto analyse visaient à :

- Présenter et discuter avec les participants les premiers résultats de l'étude "Maternité sans risque et survie de l'enfant" après la phase enquête.

- Appuyer les participants dans l'identification et la planification des actions à base communautaire à entreprendre pour résoudre les problèmes liés à la maternité sans risque et à la survie de l'enfant.

Pour obtenir l'implication maximum des participants, des méthodes actives d'animation ont été utilisées tout le long de ces ateliers. Ces méthodes sont entre autres les travaux de groupe, le brainstorming, les exposés suivis de discussions. Les ateliers étaient animés en langue locale Mooré.

La plus grande difficulté rencontrée dans la tenue de ces ateliers était liée à la saison pluvieuse, néanmoins il y avait une forte participation lors des deux ateliers. Sur environ cinquante (50) personnes invitées, la moyenne de participation à ces ateliers étaient de 39 personnes (32 participants Komsilga et 46 à Rakaye). Le public concerné était les membres des comités de gestion, les ASC, les agents SBC, les tradipraticiens, le personnel de santé des formations sanitaires concernées et les équipes cadre de district.

Les données recueillies lors de l'enquête de terrain ont été confrontées par celles obtenues des ateliers d'auto-analyse. II s'est avéré que les problèmes recueillis sur le terrain ont été mentionnés encore par les participants aux AAA. Cela a conforté l'équipe d'encadrement. Pour l'élaboration des plans d'action, on a procédé à une synthèse desdits problèmes par site et par étape de la maternité.

\section{Problèmes identifiés à Komsilga}

\section{Pendant la grossesse}

Le premier point qui revient le plus souvent est la quantité de travail dévolu à la femme (labour, pilage des céréales, port de lourd charge, coupe de bois, cueillette, corvée d'eau, etc.). Pour les participants à l'AAA, plus la femme travaille plus elle court le risque de voir sa grossesse se compliquer. Au nombre des signes précurseurs de complication les plus souvent évoqués, il y a les maladies pendant la grossesse (anémie et vomissement). Lorsque la femme n'arrive pas à satisfaire les prescriptions médicales et à avoir une alimentation suffisante et de qualité, elle court le risque de connaître des complications au cours de sa maternité. La précocité de la grossesse et le manque d'hygiène sont présentés aussi comme des situations entraînant des complications ultérieures chez la femme. Les paroles injurieuses et les disputes (à partir du huitième mois) ont pour conséquences les difficultés d'accouchement. 


\section{Pendant l'accouchement}

L'accouchement à domicile sans assistance médicale et l'impossibilité d'honorer les prescriptions médicales (notamment par manque de moyens financiers) sont les principales raisons de complication au moment de l'accouchement. L'hémorragie de la femme et la mauvaise présentation de l'enfant caractérisent une complication au moment de la délivrance. La grande multiparité et le non suivi prénatal sont des causes de complication pendant la grossesse.

\section{Au cours du post-partum}

A ce stade, c'est la mauvaise alimentation ou l'insuffisance alimentaire de la femme qui peuvent entraîner des complications chez cette dernière mais aussi chez le nouveau-né (manque de lait ou mauvaise qualité du lait). II faut noter que la consommation de repas est déconseillée car cela provoque un "noircissement $d u$ ventre" et des "plaies dans le ventre" de la nouvelle accouchée. Le non suivi du calendrier vaccinal (antitétanique notamment) aura des conséquences néfastes au cours du post-partum lointain. Enfin, l'impossibilité de satisfaire la prescription médicale postnatale, le manque d'hygiène et l'accouchement non assistée sont des éléments qui préfigurent des complications tant pour l'enfant que pour la femme.

\section{Problèmes identifiés à Rakaye}

\section{Au cours de la grossesse}

Les grands efforts physiques (travaux champêtres, coupe de bois, corvée d'eau) faits par la femme sont les premières causes laissant présager des complications. La prise de médicaments sans avis médical, la méconnaissance des problèmes liés à la grossesse, l'insuffisance alimentaire et la mauvaise qualité alimentaire de la femme, la consommation d'aliments non conseillés (œuf, sauce de "boumdou," lait) reviennent assez souvent les causes de complication à cette étape de la grossesse. L'absence de consultation prénatale a pour conséquence immédiate ou future, des complications. Enfin, le fait d'être constamment près du feu (préparation du dolo par exemple) et la position courbée (pression sur le fœtus) sont les comportements à risque fustigés par les participants.

\section{Au cours de l'accouchement}

L'accouchement à domicile sans l'assistance de la sage-femme ou de l'accoucheuse villageoise constitue la source de complication chez la femme. L'hémorragie, la mauvaise présentation de l'enfant et l'excision (antérieure) sont des signes présageant des complications. La réticence à conduire la femme en difficulté à la FS ou chez l'accoucheuse villageoise, souvent due à un manque de moyens financiers, a pour conséquence des complications. Comme cause de difficulté d'accouchement, il est fait cas de la grande multiparité (plus de sept grossesses).

\section{Au cours du post-partum}

L'insuffisance alimentaire et la mauvaise alimentation de la femme ont des conséquences néfastes chez elle comme chez l'enfant. La consommation de repas froids par la femme est déconseillée : "cela noircit son ventre." A ce stade il est reconnu que la vaccination, les soins médicaux et l'hygiène alimentaire et corporelle (mère et nouveau-né) sont déterminants pour éviter les complications. 
Certaines questions, au regard de leur importance, ont suscité de longues discussions. Ainsi :

Points d'achoppement des AAA :

- Implication des époux

- Grands efforts physiques

Les participants ont recommandé que les maris - Absence du personnel de santé soient impliqués dans la mobilisation des - Prise en charge des ASC femmes et jeunes filles pour les actions de mobilisation et de causerie ; cela constitue pour eux une condition indispensable à la durabilité des activités identifiées. Les participants ont insisté sur la complexité du problème lié aux durs travaux effectués par les femmes enceintes. Selon les hommes, "les femmes enceintes travaillent dur à cause de la famine mais aussi parce qu'elles ne connaissent pas les risques." Par exemple si les maris les autorise à se reposer, "elles iront travailler ailleurs" (dans leur champ personnel) ; "elles ne comprennent pas que c'est parce qu'elles ont "changé" qu'on les autorise à se reposer."

Pour les hommes, il y a une insuffisance ou un manque de connaissance chez les femmes à résoudre d'urgence. Les femmes rétorquent en disant que "les hommes ne subviennent pas à nos besoins ce qui fait que nous sommes obligées de travailler dur pour avoir le nécessaire." Pour elles, "le fond du problème est purement économique." Un autre sujet de grande discussion a été l'indisponibilité du personnel de santé. La question du recours à l'accoucheuse traditionnelle en l'absence du personnel de santé et de l'accoucheuse villageoise s'est posée. Une réponse pratique a été apportée. Les agents de santé communautaire ont posé la question de leur prise en charge par les populations qui n'est pas effective. Cette insuffisance couplée à la non mobilisation des populations lors des séances de causerie qu'ils organisent favorisent le découragement et le ralentissement des activités.

Au sortir des ateliers, les constats suivants ont été faits en accompagnement des plans d'action communautaire :

1. Les actions identifiées et planifiées sont essentiellement des causeries de groupes ou à domicile, des animations, de formation et d'information (IEC) dont les responsables sont notamment les ASC, les agents SBC, et le personnel de santé ; d'où la nécessité de développer ou de renforcer leurs capacités à jouer ce rôle de formateurs et d'animateurs communautaires (évaluation des besoins de formation, conception, élaboration d'un guide ou d'un canevas d'animation communautaire à l'intention des prestataires communautaires).

2. Les ASC et les agents SBC ont aussi soulevé le manque et/ou l'insuffisance de supervision d'où la recommandation selon laquelle les membres des équipes cadres de district travaillent à la mise en place du suivi ou le renforcement d'un système de • supervision opérationnel à l'intention des prestataires ci-dessus cités.

Principales recommandations causeries de groupes ou à domicile, animation, formation, information, supervision opérationnelle, système de motivation des prestataires communautaires.

3. Le récurrent problème de la motivation des prestataires communautaires reste à approfondir pour une réalisation et une pérennisation des activités. 


\section{CONCLUSIONS ET RECOMMANDATIONS}

\section{Conclusion $\mathrm{n}^{\circ} 1$}

La communauté a une connaissance de l'existence des signes précurseurs de risque pendant et durant la grossesse, l'accouchement, et le post-partum; cependant, les causes sont souvent attribuées à des facteurs socioculturels divers et fatalistes. Les adolescents qui n'ont pas encore expérimenté une grossesse et qui n'ont pas reçu une information adéquate en la matière restent ignorants des signes précurseurs de complication au cours de la grossesse et de l'accouchement.

\section{Recommandation $n^{\circ 1}$}

- La mise en place d'un programme de services de santé de la reproduction destinés aux jeunes du milieu rural.

- Ce programme devrait être intégré et exécuté dans le cadre global de l'intégration des services, prenant en compte les besoins des jeunes en matière de santé de la reproduction.

- L'élaboration et la diffusion des messages au sein de la communauté ciblant les jeunes par la formation et l'utilisation de pairs éducateurs.

La recommandation $n^{\circ} 1$ pourrait prendre la forme d'une recherche opérationnelle, passant par la collecte de données de base suivi de l'intervention constituées par les pairs éducateurs.

\section{Conclusion $n^{\circ} 2$}

II n'existe pratiquement pas de communication au sein du couple en rapport avec la grossesse, l'accouchement. La seule forme de communication au sein du couple se limite seulement à l'annonce de la grossesse au partenaire. II n'y a pratiquement pas de participation de en ce qui concerne la grossesse et l'accouchement. Leur seule participation se limite à la provision des médicaments et des aliments.

\section{Recommandation $n^{\circ} 2$}

- Au niveau communautaire: il s'agira de stimuler ou de promouvoir la communication au sein du couple en utilisant le biais des agents SBC pour discuter avec les couples et en les invitant à aller à la formation sanitaire pour les services.

- Au niveau des formations sanitaires : le personnel devrait être formé/recyclé, de même que les FS devraient s'organiser pour recevoir les couples qui y sont référés.

Cette recommandation pourrait aussi prendre la forme d'une recherche expérimentale d'implication des hommes. 


\section{Conclusion $n^{\circ} 3$}

L'itinéraire thérapeutique est polymorphe en fonction de la cause réelle ou supposée. En effet, il implique le traitement moderne, le traitement traditionnel et religieux par les prières et des sacrifices divers. Cependant force est de reconnaître que les complications sont souvent traitées traditionnellement et c'est souvent en cas d'échec que la formation sanitaire est sollicitée. Les accoucheuses villageoises et les agents SBC sont souvent sollicités pour des conseils et des soins. C'est une opportunité à exploiter pour mener des activités par leur biais.

\section{Recommandation $n^{\circ} 3$}

- Former/recycler les prestataires communautaires pour donner des conseils en matière de survie de l'enfant et de maternité sans risque. Le contenu de cette activité sera élaboré en tenant compte des résultats de la présente étude.

\section{Recommandation $n^{\circ} 4$}

- En vue de la mise en œuvre de l'ensemble de ces recommandations et d'un plan d'utilisation effective des résultats de cette étude, il est impératif qu'une table restreinte et une dissémination communautaire soient organisées 